\title{
Lossless interface modes at the boundary between two periodic dielectric structures
}

\author{
A. V. Kavokin, ${ }^{1}$ I. A. Shelykh,,${ }^{1,2}$ and G. Malpuech ${ }^{1}$ \\ ${ }^{1}$ LASMEA, UMR 6602 CNRS, Université Blaise-Pascal, 24, avenue des Landais, 63177 Aubière, France \\ ${ }^{2}$ St. Petersburg State Polytechnical University, 29, Politechnicheskaya, 195251 St. Petersburg, Russia
}

(Received 3 August 2005; published 5 December 2005)

\begin{abstract}
Tamm states of light are lossless interface modes decaying exponentially in the surrounding media. We show that they can be formed at the boundary between two periodical dielectric structures, one having a period close to the wavelength of light and another one having a period close to the double of the wavelength. The order of layers at the interface has a crucial effect on the Tamm states. The in-plane dispersion of these states is parabolic with effective masses slightly different for TE and TM polarizations, both of the order of $10^{-5}$ of the free electron mass.
\end{abstract}

DOI: 10.1103/PhysRevB.72.233102

Surface waves are a specific type of waves that are confined at the boundary between two different media. They are used in subwavelength microscopy, molecular chemistry, cancer research, etc. ${ }^{1-3}$ The most popular kind of surface wave is a plasmon formed at the boundary of metallic and dielectric media. Recently, the possibility to organize lossless surface waves at the interface between two dielectric media has been discussed. Artigas and Torner ${ }^{4}$ have proposed to use specially designed two-dimensional photonic crystals for observation of the so-called Dyakonov modes ${ }^{5}$ localized at the surface of the crystal. The photonic structure plays the role of a uniaxial dielectric medium having controllable refractive indices for ordinary and extraordinary light modes.

In this Brief Report we propose another type of lossless interface modes, which we call optical Tamm states (OTSs) by analogy with well-known Tamm states for electrons at crystal boundaries. ${ }^{6}$ Contrary to waveguided surface modes ${ }^{7}$ and to Dyakonov modes, Tamm states remain localized for any value of the in-plane wave vector (including the zero wave-vector). OTSs are formed inside the "light cone" limited by a $k=\omega / c$ condition, where $k$ is the wave vector of light and $\omega$ is its frequency. Contrary to electronic Tamm states, optical Tamm states cannot be formed at the surface, but only at the interface between two photonic structures having overlapping band gaps. We propose a simple planar multilayer structure allowing for observation of the OTSs. Ten years ago, one of us developed a theory of Tamm states for electrons confined at the boundary of two semiconductor superlattices. ${ }^{8}$ Now we have extended the method of Ref. 8 to describe OTSs. We show that they can be formed at the interface between two periodic dielectric structures having different periods. The OTS lies in the optical stop bands of both parts of the structure. Its in-plane dispersion is parabolic with an effective mass of the order of $10^{-5}$ of a free electron mass. The splitting between TE and TM polarized Tamm states increases quadratically with the in-plane wave vector. We propose realistic multilayer structures based on the porous silicon allowing for observation of the OTSs.

Let us consider the interface between two periodical dielectric structures composed by the pairs of layers of thicknesses $a_{r}, b_{r}$ and refractive indices $n_{a}, n_{b}$ on the right-hand side of the interface and thicknesses $a_{l}, b_{l}$ and indices $n_{a}, n_{b}$ on the left-hand side of the interface [Fig. 1(a)]. The Bloch theorem allows representing eigenmodes of light in any infinite periodical structure as products of periodical Bloch amplitudes and envelope functions, which are either plane waves or real exponential functions of the coordinate. The energy bands in which the eigenfunctions of light have exponentially decaying or increasing envelopes are called stopbands in photonic structures (a complete analogy with energy gaps in solid crystals). In our model structure, two different semi-infinite periodic substructures have a common interface. Our goal is to find the modes of light localized at this interface (OTSs). Evidently, their energies should lie in the stop bands of both substructures. In each of them the OTS coincides with one of the eigenmodes of light having an envelope either exponentially increasing (in the left substruc-

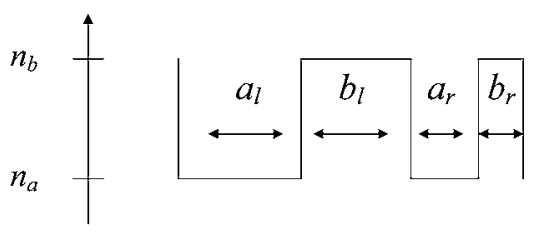

a)



FIG. 1. (a) Sketch of the interface between the right and the left parts of our model structure. (b) The solid line shows the allowed photonic band in the right substructure. The dashed line shows the allowed photonic band in the left substructure. The energies are shown of the pseudowave-vector $q$ expressed in $\pi / d$ units, $d=a_{l}$ $+b_{l}$ for the left structure, and $d=a_{r}+b_{r}$ for the right structure. The flash shows the position of the OTS which is located within the gaps of the two substructures. 
ture) or exponentially decreasing (in the right substructure). At the interface, continuity of the in-plane components of the electric and magnetic fields of these modes must be assured (as the Maxwell boundary conditions require).

Following Born and Wolf, ${ }^{9}$ we introduce the transfer matrices $T_{L}$ and $T_{R}$ describing the transfer of the vector $\left[\begin{array}{c}E_{\tau} \\ H_{\tau}\end{array}\right]$ across the periods of the left and right substructure, respectively. Here $E_{\tau}$ and $H_{\tau}$ are in-plane components of the electric and magnetic field. The elements of these $2 \times 2$ matrices are dependent on the thicknesses and refractive indices of the layers, frequency, in-plane wave vector, and polarization of light. The dispersion equation for optical modes in periodic structures writes

$$
\cos q d=\frac{t_{11}+t_{22}}{2},
$$

where $q$ is the Bloch pseudo-wave-vector of light oriented normally to the layer planes, $d$ is the period of the structure, $t_{11}, t_{22}$ are diagonal elements of the corresponding transfer matrix. The stop bands are given by the condition

$$
\left|t_{11}+t_{22}\right|>2 \text {. }
$$

The matching of $E_{\tau}$ and $H_{\tau}$ at the interface between two structures requires

$$
\left[\begin{array}{l}
E_{\tau}^{L-} \\
H_{\tau}^{L-}
\end{array}\right]=\left[\begin{array}{l}
E_{\tau}^{R+} \\
H_{\tau}^{R+}
\end{array}\right]
$$

where the left (right) part is the eigenvector corresponding the mode decaying in the negative (positive) direction in the left (right) substructure. Let $X_{L}^{-}$and $X_{R}^{+}$be the eigenvalues of the transfer matrices corresponding to these modes, so that $\left|X_{L}^{-}\right|>1$ and $\left|X_{R}^{+}\right|<1$. Expressing the components of the eigenvectors via eigenvalues and the elements of both matrices, $t_{i j}^{L, R}$ Ref. 8, one can easily obtain from (3),

$$
\frac{X_{L}^{-}-t_{11}^{L}}{t_{12}^{L}}=\frac{X_{R}^{+}-t_{11}^{R}}{t_{12}^{R}} .
$$

Equation (4) yields the energies of the OTSs and the in-plane dispersion of these states. In order to find the OTSs, one should solve Eq. (4) within the band gaps of both parts of the structure.

What kind of structures can exhibit Tamm states? To get an idea about it, let us express electric and magnetic fields in Eq. (1) via reflection coefficients $r_{L(R)}$ for light incident from the right (left) side of the interface on the left (right) part of the structure. At normal incidence

$$
\begin{gathered}
E_{\tau}^{L-}=1+r_{L}, \quad E_{\tau}^{R+}=A\left(1+r_{R}\right), \\
H_{\tau}^{L-}=n_{b}\left(1-r_{R}\right), \quad H_{\tau}^{R+}=A n_{a}\left(r_{L}-1\right) .
\end{gathered}
$$

Within the stop bands, the reflection coefficients write

$$
\begin{aligned}
& r_{L}=\frac{t_{12}^{L} n_{a}-t_{11}^{L}+X_{L}^{-}}{t_{12}^{L} n_{a}+t_{11}^{L}-X_{L}^{-}} \equiv e^{i \varphi_{L},} \\
& r_{R}=\frac{t_{12}^{R} n_{b}+t_{11}^{R}-X_{R}^{+}}{t_{12}^{R} n_{b}-t_{11}^{R}+X_{R}^{+}} \equiv e^{i \varphi_{R}} .
\end{aligned}
$$

As our left and right substructures are semi-infinite, in the overlapping part of their stop bands $\left|r_{R}\right|=\left|r_{L}\right|=1$. Resolving Eq. (3) with use of Eqs. (5)-(7) one can obtain the equation for the eigenfrequencies of the OTSs in the following form:

$$
\frac{\tan \left(\varphi_{R} / 2\right)}{\tan \left(\varphi_{L} / 2\right)}=-\frac{n_{a}}{n_{b}}
$$

This equation is relatively easy to analyze as the phases of reflection coefficients of periodic structures are well known. ${ }^{10}$ As its right part is a finite negative number, two tangents in the left part should take values of the opposite sign but of the same order. Let us underline at this point, that in one-dimensional photonic crystals the optical gaps may appear either at the center or at the border of the Brillouin zone, i.e., at either $q=0$ or $q=\pi / d$. The phase of the reflection coefficient of the structure equals to 0 at the center of the stop band at $q=0$ and can be either 0 or $\pi$ at the center of the stop band at $q=\pi / d$ dependent on whether the first layer of the structure has a lower or higher refractive index than the second layer, respectively. In all cases, the phase is a slowly increasing function of the frequency of light. Clearly, it is impossible to have both $\varphi_{L}$ and $\varphi_{R}$ close to $\pi$, having in mind that necessarily in one substructure the first layer has the high refractive index and in the other one the first layer has the low refractive index. On the other hand, it is possible to have both $\varphi_{L}$ and $\varphi_{R}$ close to 0 . It is realized if, say, $n_{a}$ $<n_{b}$, and the gap at $q=0$ in the left substructure overlaps with the gap at $q=\pi / d$ in the right substructure.

This kind of overlap can be realized, for example, between the first stop band of a perfect Bragg mirror, having

$$
n_{a} a_{r}=n_{b} b_{r} \equiv \sigma,
$$

and the second stop band of a structure with almost twice larger layers where the Bragg condition is almost satisfied, so that

$$
n_{a} a_{l}=2 \sigma+\delta, \quad n_{b} b_{l}=2 \sigma-\delta .
$$

$\delta$ is introduced in order to open the gap at $q=0(\delta \ll \sigma) .{ }^{11}$ The photonic bands of two parts of the structure are shown in Fig. 1(b). We used the following set of parameters: $n_{a}=1.4$, $n_{b}=2, n_{a} a_{l}=384 \mathrm{~nm}, n_{b} b_{l}=437 \mathrm{~nm}, n_{a} a_{r}=95 \mathrm{~nm}$, and $n_{b} b_{r}$ $=285 \mathrm{~nm}$. The chosen values of refractive indices are easily achievable, for instance, in porous silicon structures. ${ }^{12}$ Designing the right substructure, we have slightly detuned the thicknesses of layers from those given by the Bragg condition (9) in order to tune $\varphi_{r}$, that allowed bringing the OTS closer to the center of the stop bands of both substructures.

Figure 1(b) shows by a flash the energy of the OTS in our model structure. Figure 2 shows the intensity of the electric field of this mode as a function of the coordinate. Indeed, one can see that the mode decays exponentially both in negative and positive directions from the interface. The parameter of decay of the Tamm states toward the left (right) substructure, $L_{L}\left(L_{R}\right)$, is given by the eigenvalue of the corresponding transfer matrix

$$
L_{L}=d_{L} / \ln \left|X_{L}^{-}\right|, \quad L_{R}=-d_{R} / \ln \left|X_{R}^{+}\right|,
$$

where $d_{L}\left(d_{R}\right)$ is the period of the left (right) substructure. 


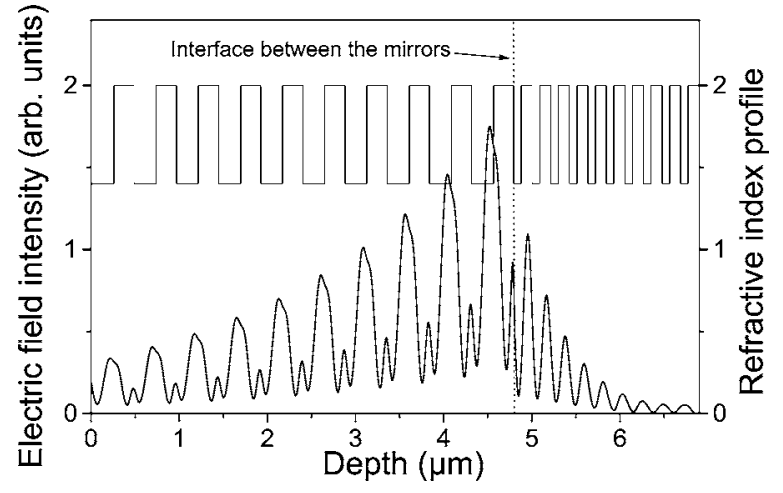

FIG. 2. The solid bold line shows the electric field intensity profile of the OTS within the proposed structure. The solid thin line is for the corresponding refractive index profile. The dotted line is for the interface between two substructures.

Interestingly, the Tamm states disappear if the order of layers is inverted [i.e., if in the left (right) structure, the layer with refractive index $n_{a}\left(n_{b}\right)$ goes first]. This can be easily understood from Eq. (8), the inversion of the number of layers changes $\varphi_{R}$ by $\pi$, while $\varphi_{L}$ remains almost constant. Clearly, the left part of Eq. (8) changes drastically in this case, and a solution can no longer be found.

At the energy of the Tamm state, the reflectivity spectrum of the structure exhibits a sharp dip inside the stop-band, as Fig. 3 shows. This calculation has been performed for a finite-size structure having 25 pairs of layers in its left part and 12 pairs of layers in its right part. This difference in the number of pairs is needed to balance the reflection coefficients of the two substructures in order to achieve the highest amplitude of the OTS feature. Changing the incidence angle pushes the Tamm state toward higher energies as one can see from the spectra in Fig. 3. Figure 4 shows the calculated in-plane dispersion of the OTSs in our structure for TE- and TM-polarized modes (i.e., modes having an electric field vector and magnetic field vector in plane of the layers, re-

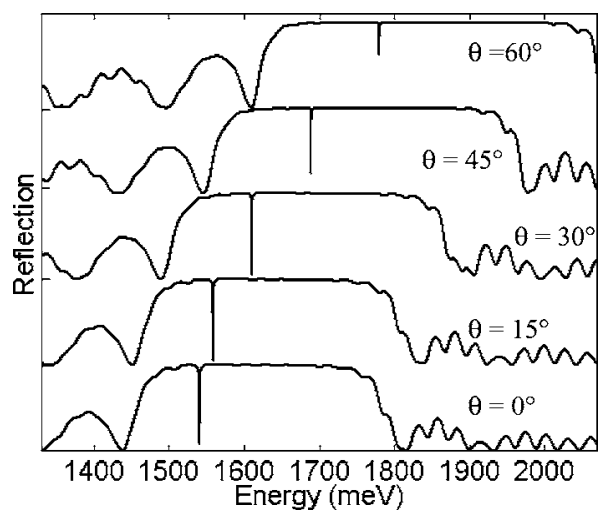

FIG. 3. Reflectivity spectra of our model structure calculated at different incidence angles. Each curve is shifted by 1 with respect to the preceding one. One can observe a dip and sharp peak within the stop band of the structure associated OTS.

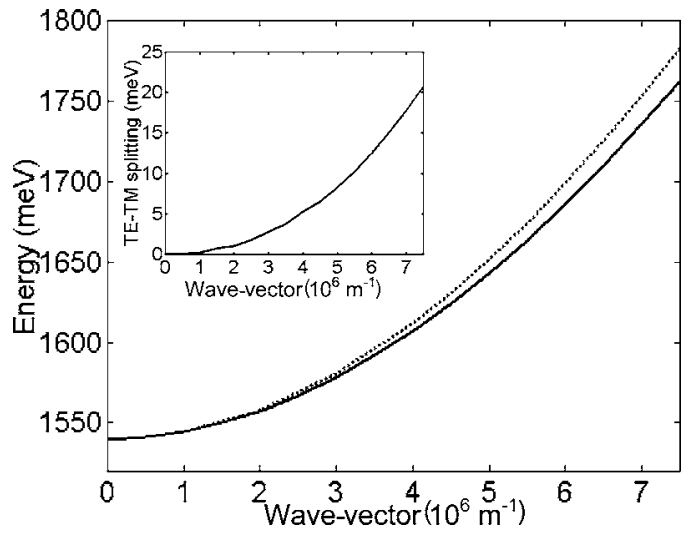

FIG. 4. Energy of the OTS as function of the in-plane wave vector in TE (solid line) and TM (dashed line) polarizations. The inset shows the energy difference between the TM and TE modes.

spectively). It is essentially parabolic in both polarizations and can be characterized by an effective mass of the order of $10^{-5} m_{0}$, where $m_{0}$ is the free electron mass. The inset in Fig. 4 shows the splitting between TM- and TE-polarized OTSs. It increases quadratically as a function of the in-plane wave vector $k$. We checked that the dispersion curves obtained solving Eq. (3) and extracted from the reflection spectra of the model structure are exactly the same.

In order to estimate analytically the effective masses of the surface modes we have expanded Eq. (2) into a Taylor series over $k$ and $\Delta \omega \equiv \omega(k)-\omega(0)$, where $\omega(k)$ is the eigenfrequency of the OTS. We assumed

$$
\omega(0) \sigma / c \approx \pi / 2 \text {. }
$$

In this case compact expressions for the effective masses in TE and TM polarizations can be obtained;

$$
\begin{gathered}
m_{e f f}^{T E} \approx \frac{\pi n_{b} n_{a}}{2} \frac{\hbar}{c \sigma}, \\
m_{e f f}^{T M} \approx \frac{\pi n_{b}^{2} n_{a}^{2}\left(n_{b}+n_{a}\right)}{2\left(n_{b}^{3}+n_{a}^{3}\right)} \frac{\hbar}{c \sigma} .
\end{gathered}
$$

$m_{\text {eff }}^{T E}$ is about $5 \%$ heavier than $m_{\text {eff }}^{T M}$ in our case. The values are very close to those which can be extracted from Fig. 4, even though our right substructure is not exactly Braggarranged.

In conclusion, optical Tamm states are a valuable alternative to the Dyakonov surface modes for realization of lossless localized photonic states in dielectric structures. They appear at the interfaces between conventional periodic multilayer structures and do not require in-plane modulation of the dielectric constant. We have proposed a method of designing the structures suitable for observation of the Tamm states. Interestingly, the OTS is extremely sensitive to the order of layers at the interface. In-plane dispersion of an OTS is parabolic with effective masses lighter than the free electron mass by a factor of $10^{5}$.

This work has been supported by the Marie-Curie RTN "Clermont2" (Contract No. MRTN-CT-2003-503677). 
${ }^{1}$ Near-Field Optics and Surface Plasmon Polaritons, edited by S. Kawata (Springer, Berlin, 2001).

${ }^{2}$ M. M. Bask, M. Jaros, and J. T. Groves, Nature (London) 427, 304 (2002).

${ }^{3}$ N. Kedei, D. J. Lundberg, A. Toth, P. Welburn, S. H. Garfield, and P. M. Blumberg, Cancer Res. 64, 3243 (2004).

${ }^{4}$ D. Artigas and L. Torner, Phys. Rev. Lett. 94, 013901 (2005).

${ }^{5}$ M. I. D’yakonov, Sov. Phys. JETP 67, 714 (1988).

${ }^{6}$ I. E. Tamm, Sov. Fiz. Zhurn. 1, 733 (1932).

${ }^{7}$ See, e.g., J. D. Joannopoulos, R. D. Meade, and J. N. Winn, Photonic Crystals (Princeton University Press, Singapore, 1995), Chap. 4.
${ }^{8}$ M. R. Vladimirova and A. V. Kavokin, Fiz. Tverd. Tela (Leningrad) 37, 1178 (1995) [Phys. Solid State 37, 1178 (1995)].

${ }^{9}$ M. Born and E. Wolf, Principles of Optics (Pergamon, Oxford, 1980).

${ }^{10}$ See, e.g., G. Panzarini, L. C. Andreani, A. Armitage, D. Baxter, M. S. Skolnick, V. N. Astratov, J. S. Roberts, A. V. Kavokin, M. R. Vladimirova, and M. A. Kaliteevski, Phys. Solid State 41, 1223 (1999).

${ }^{11}$ Perfect Bragg mirrors have no gap at $q=0$.

${ }^{12}$ V. Agarwal, J. A. del Rio, G. Malpuech, M. Zamfirescu, A. Kavokin, D. Coquillat, D. Scalbert, M. Vladimirova, and B. Gil, Phys. Rev. Lett. 92, 097401 (2004). 